\title{
LA INDUSTRIA DEL CALZADO EN LA PROVINCIA DE ALICANTE: CARACTERÍSTICAS DE SU EVOLUCIÓN RECIENTE (1970-1991)*
}

\author{
Rafael Sebastiá Alcaraz
}

\begin{abstract}
RESUMEN
Las importantes transformaciones generadas como consecuencia de los acontecimientos políticos, sociales, de los avances tecnólogicos y/o de las exigencias de los mercados, han influido en la evolución de las estructuras productivas y de los espacios geográficos industriales. En este caso la investigación se ha centrado en Alicante, y en un sector: el del calzado, que durante este período ha estado definiendo el desarrollo industrial de la provincia.
\end{abstract}

Palabras claves: Alicante, localización industrial, calzado.

\begin{abstract}
The important transformations generated as a result of the political and social events, of the technological advances and/or the requirements of the markets, have had an influence on the development of the productive structures and of the industrial geographic space. In this case the investigation has been located in Alicante, and in one sector: that of footwear, which, during this period, has been defining the industrial development of the province.
\end{abstract}

Key words: Alicante, localitation industrial, footwear.

\section{Introducción}

Resulta difícil encontrar un ejemplo de industria endógena que haya contribuido de igual modo al desarrollo económico de una región, y en concreto al de la provincia de Alicante, como es el caso de la del calzado. Según las estadísticas oficiales, este sector junto con uno de sus inputs, el cuero, durante 1991 significó en la Comunidad Valenciana

* Investigación realizada dentro del Proyecto G.V.-2401/94. 
una producción bruta valorada en 194.200 millones de ptas. (44’2\% del total español del calzado y el 6’4\% del total de la P.I.B. en la Comunidad Valenciana), y empleó a 20.591 personas (el 40'7\% del sector calzado en el Estado y el 8'2\% del total en la Comunidad). Pero donde se observa mejor una de sus peculiaridades, es en las exportaciones, ya que las ventas al exterior supusieron el 55\% de las realizadas en la provincia de Alicante, el $12 \%$ de la Comunidad y el 2\% de las de España. Dentro de la región, el sector calzado sólo fue superado en la actividad industrial por el sector material de transporte a causa del impacto de una multinacional del automóvil.

El sector sigue las pautas generales de la industria valenciana, como es el dedicarse a la elaboración de bienes de consumo de media y escasa duración, utilizar inicialmente mucha mano de obra y poco capital, junto a la aludida vertiente exportadora de la que depende en fuerte medida, su porvenir. A la primitiva escasa demanda de capital y abundante mano de obra, además con experiencia, se puede añadir otra característica como es el minifundismo empresarial.

La situación actual arranca del Plan de Estabilización de 1959 y del Programa de Ayuda a la Industria suscrito entre España y los EE.UU. El Take-off del sector se sitúa en la década de los sesenta, cuando las condiciones previas existentes, como pequeños núcleos de artesanos, abundante mano de obra, comunicaciones, etc., se unieron a la coyuntura política interior del país y a la demanda exterior procedente del mercado norteamericano. El interés de importantes sectores económicos de los EE.UU. en colocar su maquinaria y capital contribuyó definitivamente al desarrollo y a la mecanización de la actividad del calzado.

En la década de los setenta se producen una serie de problemas tanto internos de producción (incremento de los costes laborales, fiscales, materias primas) como externos (irrupción de nuevos países industriales, desarrollo del proteccionismo, depreciación del dólar) que conducen al sector hacia la crisis.

En el caso de la industria del calzado, la Administración elaboró y aprobó un Plan de Reconversión para encontrar una salida a la crisis ${ }^{1}$, cuyas líneas básicas de actuación fueron realizar planes de inversión para racionalizar la producción, aumentar la productividad como mínimo un $20 \%$, fomentar la exportación y mantener el empleo. Los resultados del Plan fueron mínimos y según el Libro blanco de la Reindustrialización fallaron, entre otras razones, porque el Plan incorporaba grandes dosis de imprecisión.

La economía sumergida, que alcanza importancia especial en la provincia de Alicante y en el sector calzado, es otra característica a tener en cuenta con el objeto de evitar las desviaciones estadísticas y las conclusiones erróneas ${ }^{2}$. Se coincide, dadas las circunstancias, en las dificultades que presenta su cuantificación, tal y como apunta Moreno $^{3}$, sin embargo existen divergencias a la hora de valorar su impacto. Por un lado, se coincide con la posición de Sintes ${ }^{4}$ cuando afirma que los «talleres piratas... afectan muy negativamente los legítimos intereses de los industriales que satisfacen sus obligaciones tributarias». Esta crítica se amplía no sólo por la competencia desleal para el empresario sino también para el trabajador, y por el expolio de los fondos públicos destinados a fines sociales, como el

1 R.D. 1.002/1982, de 14 de mayo.

2 SANCHIS GÓMEZ, Enric: «Industria subterránea y crisis económica en el País Valenciano, el final de un modelo de crecimiento», Información Comercial Española, nº 584, pp. 119-130, julio 1982.

3 MORENO FONSERET, Roque: La crisis económica en la Provincia de Alicante 1973-1983, CAPA, Alicante, 1989.

4 SINTES OBRADOR, Fernando: «El calzado alicantino en la encrucijada», Información Comercial Española, n 586, pp. 111-117, 1982. 
paro, etc.; la justificación de este tipo de economía únicamente se puede realizar por falta de conciencia social ${ }^{5}$. Por otro lado, desde el punto de vista económico y geográfico se plantea la posibilidad de que la competencia existente entre los distintos sectores y «distritos industriales», en función de los beneficios no se haya desarrollado en igualdad de condiciones y se haya alterado así la distribución espacial de la industria.

\section{La localización geográfica del sector del calzado}

La industria del calzado se caracteriza por una importante concentración espacial en pocas comarcas: Baix Vinalopó, El Vinalopó Mitjà y el Alto Vinalopó. La provincia de Alicante concentraba en 1970, el 78'7\% de las empresas, el 68' $1 \%$ del empleo y el $70 \%$ de la producción regional. La localización de esta industria dentro de la $\mathrm{CE}$ en países como Francia o Italia presentaba unas características similares a las de España ${ }^{6}$. En 1990, el sector calzado y cuero en la Comunidad Valenciana tenía 1.671 empresas en las que trabajaban 21.420 personas, lo que equivalía al $64 \%$ del total nacional en el primer caso y al $56 \%$ en el segundo. En la provincia de Alicante en esta fecha se localizaban el $95 \%$ de las empresas de la región y el 59\% de las españolas.

Los principales productores mundiales en 1990 eran China (21'8\%), la CE (11'7\%), la URSS $\left(11^{\prime} 2 \%\right)$, Taiwan $\left(7^{\prime} 1 \%\right)$ y Brasil $\left(6^{\prime} 4 \%\right)$. Europa disponía de aproximadamente unas 16.000 empresas de las cuales se localizaban en España 2.593, y en la Comunidad Valenciana $1.671^{7}$.

Además se produce una distribución de especialidades en las comarcas alicantinas. De este modo la tradición del calzado en cáñamo y esparto influye en que en Elche y su comarca se localice el calzado realizado con gran cantidad de caucho y plástico. La zona de Elda se ha especializado en calzado de señora y la de Villena, a partir de la experiencia en el trabajo con el cuero, en el calzado de niño. Las razones de esta localización se han atribuido, sobre todo, a la presencia de mano de obra especializada, a la tradición que enlaza con la existencia local de una materia prima (por ejemplo el esparto), al conocimiento del mercado, y a la facilidad de acceso para la información sobre nuevas materias primas, máquinas, clientes, etc., que permite un paso natural como es el Valle del Vinalopó, recorrido por la N-330 que une Madrid con Alicante.

Los movimientos centrífugos han contribuido desde finales de los setenta a la dispersión de las instalaciones industriales. Uno de los primeros en describir el fenómeno fue J. Ma Bernabé Maestre, hacia $1976^{8}$, posteriormente, a inicios de los ochenta A. Martínez

5 POVEDA BLANCO, Francisco: «Fraude fiscal y economía sumergida», Estructura Económica de la Provincia de Alicante, Diputación Provincial, Alicante, 1993.

«Tanto el fraude como la economía sumergida, constituyen una quiebra de la legalidad y un manifiesto atentado a los principios de equidad, igualdad y libre competencia, y si su supervivencia a corto plazo es un medio de soslayar los problemas presentes, a medio y largo plazo pueden suponer una auténtica gangrena o necrosis para el tejido empresarial».

6 Dirección General de Economía, Libro blanco sobre las repercusiones en la economía valenciana de la Adhesión de España a las Comunidades Europeas, Conselleria de Economía y Hacienda, Valencia, 1986.

7 DUARTE CARBALLO, Agustín: Informe de las principales industrias manufactureras alicantinas: el sector calzado, mármol, juguete y textil, COCIN. Alicante y Universidad de Alicante, FUNDEUN, Alicante, Junio 1992.

8 BERNABÉ MAESTRE, J.M.: La industria del calzado en el Valle del Vinalopó, Dpto. de Geografía, Universidad de Valencia, 1976. 
Gómez ${ }^{9}$ vuelve a describir esta situación. En 1990 Dávila y Ponce abordan el tema de la dispersión de esta industria por las zonas rurales del valle del Vinalopó ${ }^{10}$.

La concentración del sector es un hecho evidente, y más en sus núcleos vertebradores, Elche y Elda, pero a su alrededor se ha producido una difusión sobre municipios con economía agrícola o con alguna industria, también de carácter endógeno y con frecuencia asociada a la transformación de materias primas locales. En algunos casos, como en el de los municipios agrícolas, se ha producido un alto grado de concentración de la actividad industrial en el calzado, en otros el grado de concentración dependerá de los beneficios y de la coyuntura de los otros sectores previamente establecidos. La competencia espacial y sectorial se observa en municipios como Aspe, Monóvar, Novelda, Pinoso o Sax donde existía o existe una relevante industria de la madera, alimentación o extractiva del mármol, ampliable a otros espacios estructurados en torno a la industria del juguete o textil.

Finalmente, la concentración geográfica de las empresas del sector, en opinión de los gerentes, es altamente beneficiosa, sobre todo por las economías de aglomeración, mientras las desventajas que conlleva, como son la competencia en la distribución de mercancías o las procedentes del abastecimiento de materias primas, no son importantes ${ }^{11}$.

\section{Características del sector del calzado}

\section{El tamaño de las empresas}

Desde 1970 a 1991 el número de empresas se redujo en la Comunidad Valenciana un 42`3\% y el empleo un 61,3\%, el resultado fue un aumento del minifundismo: 20 '5 empleos por empresa en 1970 y 13,7 en 1991 (cuadro I). En este sentido también se puede señalar que en 1990 sólo quedaba una empresa con más de 100 trabajadores en toda la Comunidad Valenciana.

El origen artesanal y el escaso capital que se requería para la instalación de una fábrica influyeron en el pequeño y mediano tamaño de las empresas. La legislación laboral, que perjudicaba a las empresas con más de 50 trabajadores, también contribuyó inicialmente al modelo minifundista, aunque posteriormente sin esta traba ha continuado aumentando el número de PYMEs.

El tamaño también se relaciona con el alto grado de especialización de las empresas del calzado en las distintas fases del proceso productivo. La descentralización al modo italiano — que dejaba para las empresas de calzado el montaje y comercialización de la producción, quedando para los talleres auxiliares el resto del trabajo- se introdujo a mediados de los setenta en las empresas alicantinas, y se relaciona igualmente con el predominio de los pequeños y medianos establecimientos. En Italia, país competidor en el sector, la estructura era similar a la nuestra a finales de los ochenta, con un predominio de las pequeñas instalaciones (el $84 \%$ de las empresas no llegaban a 20 empleados y sólo el 1,5 superaba $\left.\operatorname{los} 100^{12}\right)$.

9 MARTÍNEZ GÓMEZ, A., en el Libro blanco sobre las repercusiones en la economía valencia de la Adhesión de España a las Comunidades Europeas, Conselleria de Economía y Hacienda, Valencia, 1986.

10 DÁVILA LINARES, J.M. y PONCE HERRERO, Gabino: «El proceso de industrialización rural de un área periférica: El valle del Vinalopó (Alicante)», III Reunión de Geografía industrial, Sevilla, Septiembre, 1990.

11 DUARTE CARBALLO, Agustín: Informe de las principales industrias manufactureras alicantinas: el sector calzado, mármol, juguete y textil, COCIN. Alicante, y Universidad de Alicante, Fundeun, Junio 1992.

12 DALMAU PORTA, Juan Ignacio; DE MIGUEL FERNÁNDEZ, Enrique y MIQUEL PARIS, Salvador: Análisis estratégico de los sectores industriales y del turismo en la Comunidad Valenciana, 2 vol., Fundación de Estudios Bursátiles y financieros, Universidad Politécnica de Valencia, Valencia, 1993. 


\section{Cuadro I}

LA INDUSTRIA DEL CALZADO EN LA COMUNIDAD VALENCIANA

\begin{tabular}{|lrrrrr|}
\hline Año & $\begin{array}{c}\text { Producción } \\
\text { Bruta }\end{array}$ & $\begin{array}{c}\text { Valor } \\
\text { Añadido }\end{array}$ & $\begin{array}{c}\text { Personas } \\
\text { Ocupadas }\end{array}$ & $\begin{array}{c}\text { Costes } \\
\text { Personal }\end{array}$ & Empresas \\
\hline 1970 & 16.884 & 6.865 & 40.675 & 4.374 & 1.987 \\
1971 & 22.565 & 9.081 & 43.140 & 6.059 & 1.990 \\
1973 & 33.121 & 13.229 & 49.058 & 8.727 & 1.902 \\
1975 & 47.479 & 20.285 & 49.700 & 13.099 & 2.019 \\
1977 & 71.489 & 27.323 & 51.494 & 21.879 & 1.951 \\
1980 & 79.976 & 34.743 & 34.990 & 25.852 & 1.636 \\
1981 & 82.400 & 32.760 & 29.630 & 25.487 & 1.499 \\
1982 & 78.814 & 31.912 & 23.660 & 22.858 & 1.095 \\
1983 & 85.381 & 32.242 & 20.537 & 22.304 & 915 \\
1984 & 113.180 & 42.800 & 21.309 & 26.096 & 1.035 \\
1985 & 111.166 & 40.217 & 19.261 & 25.690 & 1.010 \\
1986 & 113.403 & 42.632 & 18.239 & 25.075 & 1.054 \\
1987 & 104.999 & 38.489 & 17.038 & 23.083 & 1.153 \\
1988 & 123.521 & 44.504 & 16.737 & 23.145 & 1.249 \\
1989 & 138.633 & 46.920 & 18.474 & 26.121 & 1.443 \\
1990 & 153.503 & 48.250 & 17.024 & 27.207 & 1.236 \\
1991 & 152.486 & 45.704 & 15.740 & 26.122 & 1.145 \\
\hline
\end{tabular}

(1) Los valores de producción, VAB y Costes laborales, son en millones de pts.

Servicio Sindical de Estadística, Estadística de producción Industrial, Madrid, varios años. (Hasta 1977). IVE, Resultats de l'Enquesta industrial de l'INE per a la Comunitat Valenciana, Valencia, varios años.

La parquedad de las plantillas de los establecimientos también depende de forma trascendente de la demanda de un mercado irregular, de la moda y de la necesidad de ser flexibles y sensibles a estos cambios.

\section{Duración de las empresas}

Como se ha expuesto en la obra de Hernández y Alcaraz ${ }^{13}$, la crisis industrial de los años ochenta se tradujo en cierre para muchas empresas y su paso a la economía sumergida. Los posteriores incentivos a la creación de empresas y contratación de trabajadores, han influido en que muchos de estos talleres clandestinos aflorasen a finales de los ochenta; por lo que la fecha administrativa de estas empresas apenas superaba los cinco años como se puede comprobar en DIRNOVA $92^{14}$. En una evolución «normal», la mayoría de las empresas debería tener fecha de fundación entre 1960 y 1970, coincidiendo con el boom del sector, por lo que en 1991, gran parte de éstas, contarían con más de 20 años de funcionamiento.

13 HERNÁNDEZ PASCUAL, Clemente y ALCARAZ GARCÍA, Enrique: Cambios en el tejido empresarial de la Provincia de Alicante, Fundesem, Alicante, 1989.

14 I.M.P.I.V.A.: Directorio de empresas innovadoras de la Comunidad Valencia, Generalitat Valenciana, Valencia, varios años. 


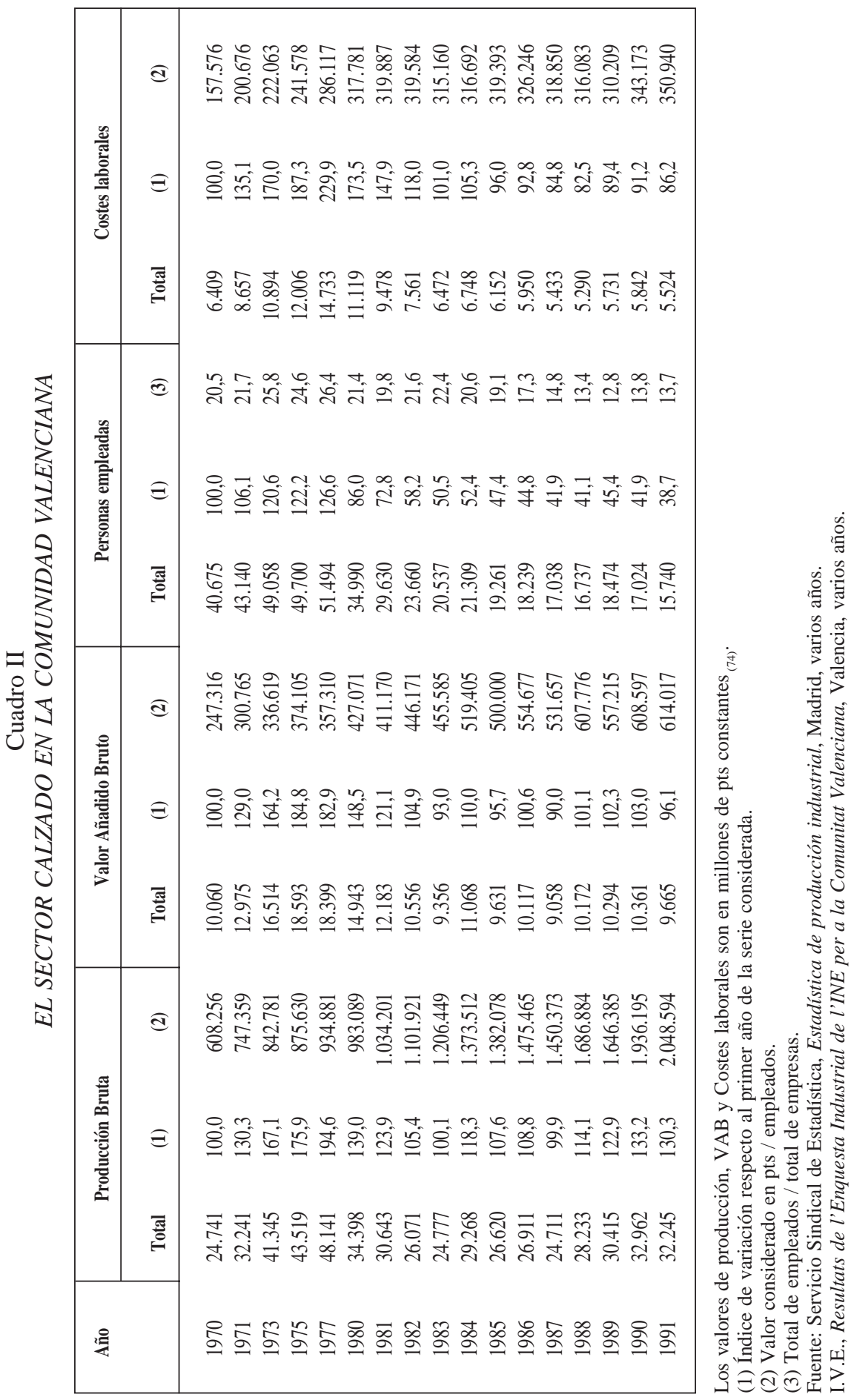


Durante la década de los sesenta y setenta el sector del calzado demandó mucha mano de obra. Así entre 1970 y 1977 el número de personas ocupadas en la Comunidad Valenciana aumentó un $26^{\prime} 6 \%$ pese a que las empresas se redujeron un $1{ }^{\prime} 8 \%$; era la etapa fordista. La producción (pts. constantes) consiguió el máximo del período en 1977, sin embargo su futuro se hallaba hipotecado por el incremento de los costes salariales que pasaron de ser el 26\% de la producción en 1970 al 30'6\% en 1977 (cuadro II).

Desde 1977 se inició la crisis en las exportaciones y las empresas se encontraron con plantillas sobredimensionadas ${ }^{15}$. El incremento de los costes laborales, de las materias primas y de la energía, contribuyeron a agravar la economía de las empresas.

A partir de 1978 se distinguen dos etapas en la evolución del sector:

La primera (1978-83) corresponde a una reducción acusada, a la par, tanto del empleo como del número de empresas. Esta etapa ofrece uno de los momentos en que mayor desarrollo adquiere la economía sumergida ${ }^{16}$. Mientras tanto los costes laborales continuaron siendo elevados, próximos al $30 \%$ de la producción.

La segunda etapa (1983 a 1991) recoge los efectos de la incorporación de España a la C.E., caracterizándose por una reducción del empleo $(23,4 \%)$ y un aumento del número de empresas $(25,1 \%)$. En esta etapa se inicia el descenso progresivo de los costes laborales; éstos en 1984 representaban el 23\% de la producción y en 1991 el 17'1\%.

En 1991 los empresarios todavía consideraban que el coste de la mano de obra era su principal problema dentro de las fases productivas ${ }^{17}$. Durante estos años la economía sumergida ha resultado nociva porque ha descapitalizado las empresas y no se ha preocupado de los diseños, ni de la calidad, ni de la innovación. Tampoco ha podido asumir costes como publicidad o salarios de mano de obra cualificada y ha obstaculizado la libre competencia.

Por otro lado, a la mano de obra se le pretendía exigir una formación que la Formación Profesional no le daba y el empresario tampoco podía ni se hallaba dispuesto a remunerar según cualificación, especialmente en los casos de Técnicos Medios y Superiores. La flexibilidad en la duración de los contratos de la mano de obra fue otra particularidad del sector vinculada a la oscilación de la demanda, y cuyos costes sociales asumió el trabajador.

La productividad por trabajador y empresa vista en relación a la Producción Bruta en pesetas constantes $_{(74)}$ se incrementó entre 1970 y 1991 (cuadro II).

La productividad por trabajador en relación al V.A.B. y en pesetas constantes ${ }_{(74)}$ siguió una evolución ascendente durante el mismo período.

En cuanto a las interralaciones con otros sectores, la concentración de la producción en la provincia contribuyó a que el 50\% de los inputs intermedios se obtuviesen en ésta: el sector del cuero y curtición aportaron el $33 \%$ de los mismos, y el químico, plástico y caucho el $19 \%$.

El cuero, una de las materias primas más importantes que intervienen en el calzado, procedió en gran parte del extranjero ya que la producción nacional era deficitaria para el consumo nacional; dentro de la demanda de cuero existía una especial preferencia por las

15 SINTES OBRADOR, Fernando: «El calzado alicantino en la encrucijada», I.C.E., n ${ }^{\circ}$ 586, 1982.

16 MARTÍNEZ GÓMEZ, A.: La política de reestructuración y reconversión en España, El proceso de reestructuración del sector calzado, Tesis de licenciatura FCEE y EE, Universidad de Alicante.

17 DUARTE CARBALLO, Agustín: Informe de las principales industrias manufactureras alicantinas: el sector calzado, mármol, juguete y textil, COCIN. Alicante, y Universidad de Alicante, Fundeun, Junio 1992. 
pieles pequeñas españolas a causa de su mayor calidad. Esta dependencia era problemática, además, porque en períodos de expansión del sector los suministros de pieles no crecían con la misma rapidez y se producía un encarecimiento de la materia prima. Por otro lado, los países productores de pieles frenaban la exportación, con la finalidad de favorecer su transformación y añadir valor a sus exportaciones. En relación con la producción de cuero cabe señalar que la Comunidad Valenciana era la segunda productora de curtidos tras Cataluña ${ }^{18}$.

Las materias primas de tipo químico (caucho y plástico) se importaban en gran parte por empresas ubicadas en Madrid y Cataluña desde países como Alemania o Inglaterra; en la provincia de Alicante se localizaba una pequeña industria química de Caucho y Plástico dependiente del sector.

Una solución que se pensó para evitar la dependencia de las materias primas fue la de fundar una central de compras, asesorados por la Universidad de Alicante ${ }^{19}$.

El coste de las materias primas, su calidad y la facilidad para obtenerlas, desde el punto de vista empresarial era considerado «como poco problemático», en entrevistas realizadas durante $1991^{20}$.

El coste de la tecnología es otro elemento a tener en cuenta en los procesos productivos e incremento de la producción y de la productividad, tanto de los establecimientos como de los trabajadores. Ya casi de forma «tradicional» existía una importante industria auxiliar y de componentes del calzado que se dedicaba a fabricar hormas, pisos, tacones, tapas, contrafuertes, plantillas que se realizaban en pequeños talleres y que se sitúan en el plano de la descentralización del proceso industrial. Estas empresas facilitaban parte de la tecnología que el sector requería, pero era evidente la dependencia del mercado internacional. La maquinaria en su mayor parte se importaba de Italia, y en menor proporción de Alemania, Inglaterra, Dinamarca y América. No existían muchas empresas españolas capaces de atender esta demanda, muchas de ellas se limitaban a copiar los modelos exteriores. Esta deficiencia se intentó superar con la creación del Instituto Español de Calzado y Conexas (INESCOP). Entre el 60 y $70 \%$ de la maquinaria empleada en el sector se calcula que era importada. Las empresas españolas de maquinaria se localizaban mayoritariamente en Alicante (Elche, Elda y Villena); en Almansa, Barcelona, Zaragoza, Vigo, Pamplona y Vitoria se hallan casi todas las restantes.

En 1991 el sector del calzado, pese a estas industrias españolas y al esfuerzo público, todavía dependía de Italia y Alemania, países desde donde se continuaba importando la maquinaria. Pero la cuestión tecnológica se había agravado por la introducción de la informática y por la reducción de los beneficios que dificultaban su adquisición. No obstante los empresarios, en encuestas y entrevistas, reconocían que en los últimos años habían continuado incorporando nuevas tecnologías.

Por último, la productividad del sector, comparada en el espacio europeo y en especial con su principal país competidor que es Italia, se puede considerar que es alta en cuanto a producción y facturación por empleo, pero el problema está en que la producción es de media y baja calidad, por lo que se pierde esta ventaja inicial ${ }^{21}$.

18 Libro blanco sobre las repercusiones en la economía valenciana de la Adhesión de España a las Comunidades Europeas, Ob. Cit.

19 «Según las noticias recogidas en nuestras visitas, se viene pensando, con la colaboración de la Universidad de Alicante, en la viabilidad de una central de compras. A pesar de su dificultad, sería un factor más para reducir la fuerza de los proveedores» DALMAU PORTA, Juan Ignacio; DE MIGUEL FERNÁNDEZ, Enrique y MIQUEL PARIS, Salvador: Ob. cit.

20 DUARTE CARBALLO, Agustín: Ob. cit.

21 CÁMARA OFICIAL DE COMERCIO, INDUSTRIA Y NAVEGACIÓN DE ESPAÑA: Informe Peat Marwick, Noviembre de 1992. 
Como conclusión se puede afirmar a inicios de los noventa que la situación del sector era de tránsito entre una industria que competía a base de costes inferiores, en especial de la mano de obra, a otra que buscaba un producto de calidad y diseño, que incorporase las nuevas tecnologías y que permitiese compensar el incremento de los costes. En este cambio, los especialistas consideraban que las empresas más pequeñas estaban destinadas a convertirse en talleres auxiliares de las grandes o a desaparecer ${ }^{22}$.

\section{La demanda}

Una característica fundamental de la industria alicantina ha sido su carácter exportador; este hecho resulta relevante en el caso de la industria del calzado, pues su aportación al total de las exportaciones alicantinas supera el 50\%, y en el caso de la Comunidad, el calzado sólo fue superado, dentro de la exportación industrial y en fechas recientes, por el impacto de una multinacional de la industria automovilística.

No obstante, la evolución de las exportaciones del sector en el conjunto de la Comunidad Valenciana indica, por un lado, una reducción de la aportación del calzado a los totales, $\mathrm{y}$, por otra, una diversificación y crecimiento de otros sectores que contribuyen a que la tendencia exportadora no retroceda. Esta situación se confirma al comprobar que en 1973 la industria del calzado aportaba el 22'8\% de lo exportado por la Comunidad Valenciana mientras que en 1991 era del 10'4\%.

Las exportaciones del sector calzado en la provincia de Alicante, aunque se hallan en crisis, su aportación al conjunto provincial continúa siendo superior al 50\%. El total de las ventas exteriores alicantinas depende de la evolución de la industria del calzado y adolece de una peligrosa falta de diversificación. Por otra parte, en la Provincia de Alicante se confirma, una vez más, la concentración del sector, ya que de forma general las exportaciones alicantinas de calzado suponen más del $90 \%$ de las de la Comunidad Valenciana (cuadro III).

El valor de las exportaciones ha variado mucho no sólo por causas de la producción, sino de la política monetaria nacional e internacional y de la incorporación de nuevos países a esta actividad. En este sentido cabe apuntar que la política internacional tendió a facilitar el desarrollo de los países subdesarrollados o en vías de desarrollo, y a crear una nueva división internacional del trabajo. La política de las Naciones Unidas (Conferencia de Lima), la Distensión y la desaparición de los dos Bloques o los acuerdos del GATT fueron algunos de los ejemplos de esta tendencia, que aunque resultaban afortunadamente positivas para los países con más problemas, por otro lado aumentaban la competencia a nuestra industria e influyeron en la pérdida de mercados.

Las ventas al extranjero estuvieron controladas por grandes cadenas comerciales foráneas, que eran las que disponían de marcas y podían costear las campañas de publicidad. El pequeño y mediano fabricante, debido entre otras razones a las cortas tiradas, no pudo crear ni marca, ni moda, ni pagar la publicidad, y por consiguiente tampoco tuvo recursos para negociar el precio de venta de su producción. Los márgenes comerciales, por consiguiente, no fueron fijados por los fabricantes, sino por los vendedores que fueron quienes sacaron mayor provecho. La creación de la FICIA (Feria Internacional del Calzado e Industrias Afines) permitió paliar esta situación y también favoreció el aumento y diversificación de las exportaciones.

En el desarrollo del sector del calzado durante el período analizado, la demanda interior cada vez ha sido menos trascendente; simplemente basta observar el valor de la 
Cuadro III

LA EXPORTACIÓN EN EL SECTOR DEL CALZADO

\begin{tabular}{|c|c|c|c|c|c|c|c|}
\hline \multirow[b]{3}{*}{ Año } & \multicolumn{3}{|c|}{ COMUNIDAD VALENCIANA } & \multicolumn{4}{|c|}{ ALICANTE } \\
\hline & \multicolumn{3}{|c|}{ (Millones de pesetas corrientes) } & \multicolumn{4}{|c|}{ (Millones de pesetas corrientes) } \\
\hline & Total & Calzado & $(\%)$ & Total & Calzado & $(\%)$ & (1) \\
\hline 1973 & 51.176 & 11.687 & 22,8 & 17.845 & 10.035 & 56,2 & 85,9 \\
\hline 1974 & 58.641 & 15.026 & 25,6 & 21.184 & 14.058 & 66,4 & 93,6 \\
\hline 1975 & 75.815 & 18.465 & 24,4 & 24.041 & 16.736 & 69,6 & 90,6 \\
\hline 1976 & 96.360 & 23.423 & 24,3 & 33.254 & 22.672 & 68,2 & 96,8 \\
\hline 1977 & 147.312 & 26.786 & 18,2 & 38.202 & 24.776 & 64,9 & 92,5 \\
\hline 1978 & 181.568 & 31.917 & 17,6 & 47.136 & 29.446 & 62,5 & 92,3 \\
\hline 1979 & 223.591 & 32.466 & 14,5 & & & & \\
\hline 1980 & 250.739 & 27.916 & 11,1 & 67.047 & 25.218 & 37,6 & 90,3 \\
\hline 1981 & 316.407 & 38.552 & 12,2 & 83.515 & 34.936 & 41,8 & 90,6 \\
\hline 1982 & 352.905 & 49.992 & 14,2 & & & & \\
\hline 1983 & 449.043 & 68.141 & 15,2 & & 61.070 & & 89,6 \\
\hline 1984 & 701.628 & 97.373 & 13,9 & 169.573 & 92.290 & 54,4 & 94,8 \\
\hline 1985 & 719.984 & 111.675 & 15,5 & & & & \\
\hline 1986 & 739.860 & 104.762 & 14,2 & 173.608 & 98.608 & 56,8 & 94,1 \\
\hline 1987 & 729.790 & 103.828 & 14,2 & 187.303 & 98.015 & 52,3 & 94,4 \\
\hline 1988 & 756.976 & 101.754 & 13,4 & 182.552 & 95.822 & 52,5 & 94,2 \\
\hline 1989 & 832.922 & 102.194 & 12,3 & 185.807 & 96.214 & 51,8 & 94,1 \\
\hline 1990 & 901.843 & 116.564 & 12,9 & 198.536 & 109.268 & 55,0 & 93,7 \\
\hline 1991 & 1.011 .074 & 105.196 & 10,4 & 190.903 & 99.890 & 52,3 & 95,0 \\
\hline
\end{tabular}

(1) Calzado alicantino / Calzado en la Comunidad Valenciana.

Fuente: COCIN de Alicante, Castellón y Valencia, La Región exporta, varios años.

producción y de lo exportado para comprobar la evolución de su incidencia. Pero cuando el mercado internacional se ha estancado muchos empresarios han encontrado en el interior una válvula de escape. En 1990 las exportaciones supusieron el 75'9\% de la Producción Bruta.

El mercado interior ha presentado también importantes cambios. La elevación del nivel adquisitivo de los españoles, ha repercutido en un nuevo tipo de demanda que exige productos de mejor calidad y diseño. La apertura del mercado interior, ha permitido el acceso al mercado interior de las producciones procedentes tanto de países donde el diseño y la moda son importantes en su producción, como es el caso de Italia, como de los nuevos países industrializados, en especial los de Asia donde los costes laborales son menores. De este modo el valor de las importaciones de calzado entre 1986 y 1991 se ha multiplicado por 6'8 en España (47.172 millones de pts. en 1991), 5'4 en la Comunidad Valenciana (7.231 millones de pts. en 1991) y 5'1 en la provincia de Alicante; ésta ofrece una 

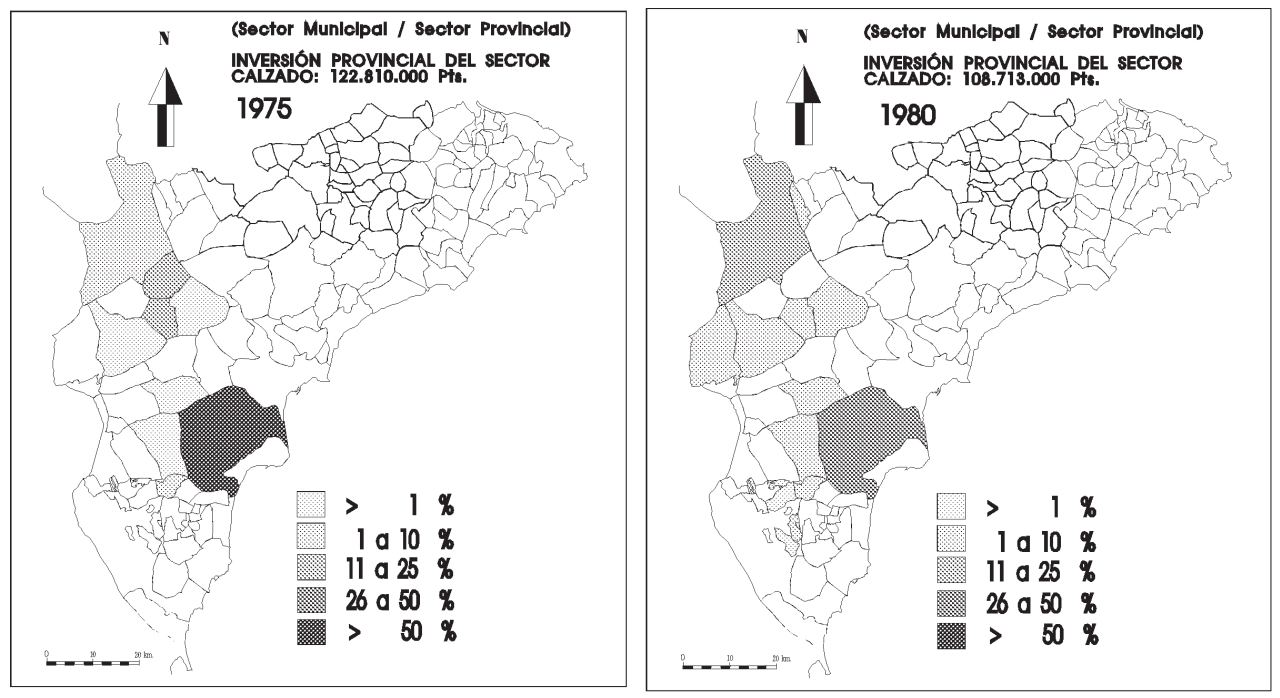

Inversión en el sector calzado en 1975, 1980, 1985, 1991
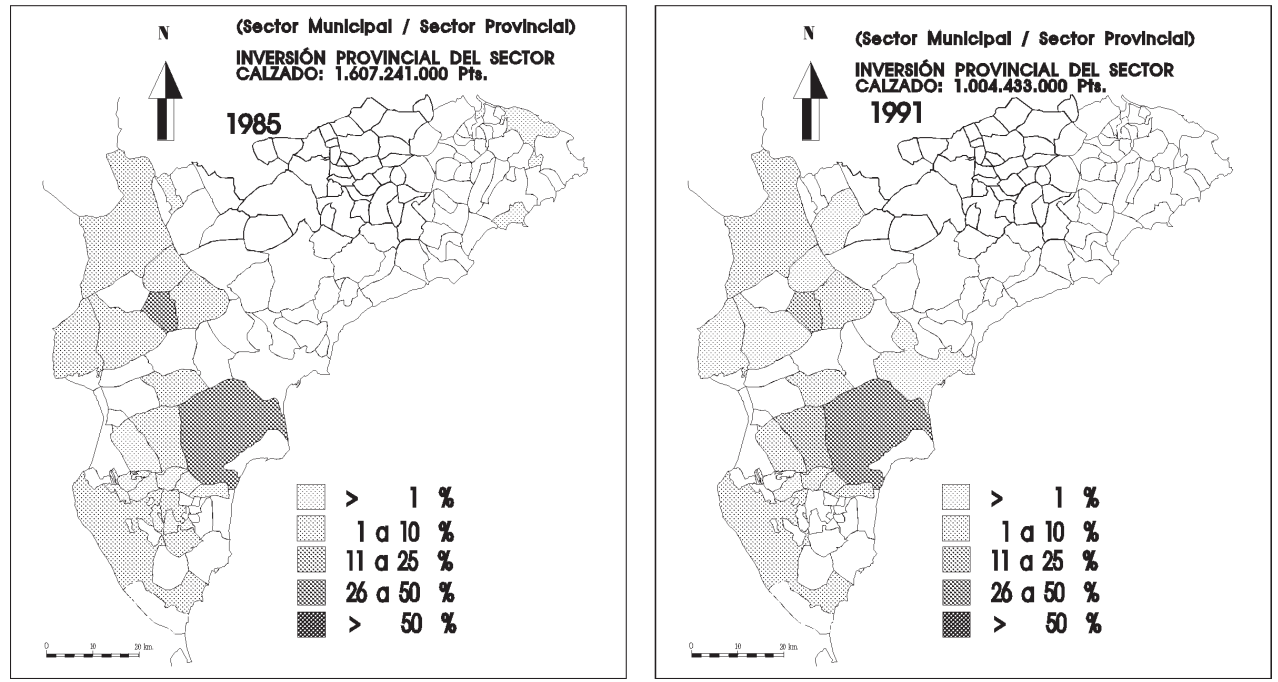

característica añadida, y es que la mayor parte de las importaciones de la Comunidad pasaron por Alicante. En 1984 el 75'6\% de las importaciones de la Comunidad entraron por Alicante y en 1991 fueron el 83'3\% ${ }^{23}$.

23 CÁMARAS OFICIALES DE COMERCIO, INDUSTRIA Y NAVEGACIÓN, DE ALICANTE, CASTELLÓN, VALENCIA, ALCOY Y ORIHUELA: La región exporta, Valencia, varios años, I.V.E.: Estadística de comerç exterior, Comunitat Valenciana, Valencia, varios años. 


\section{Inversión}

\section{Evolución de la inversión}

La evolución de la inversión en el sector calzado de la provincia de Alicante (cuadro IVa y IVb) se ha distribuido de forma irregular en función de las condiciones de producción y del mercado, aunque el resultado del período ofrece una tendencia subyacente recesiva. La industria del calzado no ha definido, como le corresponde por su empleo y número de empresas, la evolución de la inversión industrial provincial. La inversión regional del sector, al igual que las otras variables de producción, mano de obra, etc., también se halla fuertemente concentrada en la provincia de Alicante, pues esta es superior al $90 \%$ del total de la Comunidad Valenciana.

Las etapas por las que ha pasado el sector han sido:

La primera, iniciada sobre 1960, se prolongó hasta 1973 y coincidió con un período de fuertes inversiones, incremento del número de personas ocupadas y de las exportaciones.

La segunda de 1974 a 1980 se define por ser años recesivos para la inversión, donde se percibe claramente los acontecimientos políticos de 1975 en el ánimo del inversor, pero las exportaciones y la producción, aunque estancada se mantiene en los mismos niveles.

La tercera etapa se extiende de 1980 a 1986 . En ella la inversión alcanza el fondo de la crisis e inicia otra etapa de recuperación. Durante estos años se produce la segunda crisis del petróleo. De esta forma el sector fue afectado en su producción, mientras en la demanda se alcanzan los mínimos de las dos décadas analizadas. Por otra parte, el número de empresas y de personas ocupadas sufrirán uno de los descensos más fuertes. La inversión que tiene un lento crecimiento hasta 1984, se intensifica a partir de 1985, coincidiendo con el período de incorporación a la CE y alcanza casi los mismos niveles de inicio de la década de los setenta.

En la cuarta etapa, 1987 a 1991, pasados los efectos de la integración en la CE, los empresarios, al observar la evolución recesiva de la demanda, frenan el ritmo de las inversiones.

\section{Factores de los que depende la inversión}

La escasa demanda de capital que inicialmente se necesitaba para la creación de un establecimiento del sector calzado, permitió la formación de numerosas PYMEs, como se comprueba al comparar con la media del total de sectores industriales de la provincia de Alicante (cuadro V). El calzado es, pues, un sector que hasta finales de los años 1970, con una escasa inversión se creaban muchos puestos de trabajo, y de esta forma lo ven estudios de la época ${ }^{24}$. Esta cantidad disminuyó con la crisis, al desarrollarse la economía sumergida, pero con el paso del tiempo la necesidad de aumentar la productividad y la competividad de las empresas ha conducido hacia una renovación de los procesos y equipamientos productivos con lo que la inversión por inscripción y empleo se ha vuelto a incrementar. De modo que, aunque en 1991 tanto la inversión por empleo como por inscripción en el sector calzado continua siendo inferior a la media provincial de la inversión industrial total, las diferencias se han reducido ostensiblemente. Esta característica puede influir en que respecto a 1980 cada vez sea menor la capacidad de un trabajador para reunir el capital necesario y crear una empresa; o que el empresario necesite más dinero para ampliar una instalación.

24 DIRECCIÓN GENERAL DE ECONOMÍA: Ob. cit. 
Cuadro IV a

INVERSIÓN EN EL SECTOR CALZADO (MILES PTS. CORRIENTES)

\begin{tabular}{|c|c|c|c|c|c|c|c|}
\hline \multicolumn{4}{|c|}{ COMUNIDAD VALENCIANA } & \multicolumn{4}{|c|}{ ALICANTE } \\
\hline Año & Total & Calzado & $\%$ & Total & Calzado & $\%$ & (1) \\
\hline 1973 & 5.324 .303 & 388.450 & 7,30 & 1.540 .770 & 380.337 & 24,68 & 97,91 \\
\hline 1974 & 8.051 .807 & 342.117 & 4,25 & 1.934 .823 & 329.833 & 17,05 & 96,41 \\
\hline 1975 & 7.543 .721 & 152.219 & 2,02 & 2.323 .259 & 122.810 & 5,29 & 80,68 \\
\hline 1976 & 15.607 .883 & 320.158 & 2,05 & 3.133 .913 & 319.683 & 10,20 & 99,85 \\
\hline 1977 & 23.970 .516 & 342.624 & 1,43 & 2.454 .067 & 326.978 & 13,32 & 95,43 \\
\hline 1978 & 7.950 .972 & 372.962 & 4,69 & 2.564.318 & 355.562 & 13,87 & 95,33 \\
\hline 1979 & 8.127 .843 & 353.261 & 4,35 & 1.988 .215 & 335.594 & 16,88 & 95,00 \\
\hline 1980 & 4.799 .326 & 117.986 & 2,46 & 1.544 .371 & 108.713 & 7,04 & 92,14 \\
\hline 1981 & 15.902 .406 & 290.844 & 1,83 & 3.699 .696 & 263.974 & 7,14 & 90,76 \\
\hline 1982 & 15.276 .103 & 691.761 & 4,53 & 4.283 .511 & 658.686 & 15,38 & 95,22 \\
\hline 1983 & 13.253 .377 & 591.039 & 4,46 & 2.929 .612 & 548.268 & 18,71 & 92,76 \\
\hline 1984 & 32.146 .357 & 610.225 & 1,90 & 4.278 .033 & 575.890 & 13,46 & 94,37 \\
\hline 1985 & 20.970 .444 & 1.680 .446 & 8,01 & 6.867 .615 & 1.607.241 & 23,40 & 95,64 \\
\hline 1986 & 30.053 .242 & 1.731 .450 & 5,76 & 7.320 .735 & 1.534 .013 & 20,95 & 88,60 \\
\hline 1987 & 38.937 .529 & 2.041 .835 & 5,24 & 9.062 .534 & 1.914 .707 & 21,13 & 93,77 \\
\hline 1988 & 61.273 .962 & 1.630 .742 & 2,66 & 9.788 .677 & 1.540 .146 & 15,73 & 94,44 \\
\hline 1989 & 44.275 .666 & 1.043 .618 & 2,36 & 9.426 .619 & 1.005 .146 & 10,66 & 96,31 \\
\hline 1990 & 42.856 .074 & 847.497 & 1,98 & 11.676 .153 & 822.647 & 7,05 & 97,07 \\
\hline 1991 & 68.121 .059 & 1.100 .279 & 1,62 & 8.228 .233 & 1.004 .433 & 12,21 & 91,29 \\
\hline
\end{tabular}

(1) \% de inversión sectorial de la provincia de Alicante sobre el calzado de la Comunidad Valenciana.

Consellería d'Indústria, Comerç i Turisme, Inversión industrial Registrada, Valencia, varios años.

Cuadro IV b

INVERSIÓN EN EL SECTOR CALZADO (MILES PTS. CONTANTES $\left.{ }_{(74)}\right)$

\begin{tabular}{|c|c|c|c|c|c|c|c|}
\hline \multicolumn{4}{|c|}{ COMUNIDAD VALENCIANA } & \multicolumn{3}{|c|}{ ALICANTE } & \multirow{2}{*}{$\frac{(1)}{\%}$} \\
\hline Ã̃o & Total & Calzado & $\%$ & Total & Calzado & $\%$ & \\
\hline 1973 & 6.646 .359 & 484.904 & 7,30 & 1.923 .352 & 474.777 & 24,68 & 97,91 \\
\hline 1974 & 8.051 .807 & 342.117 & 4,25 & 1.934 .823 & 329.833 & 17,05 & 96,41 \\
\hline 1975 & 6.914 .501 & 139.522 & 2,02 & 2.129 .477 & 112.566 & 5,29 & 80,68 \\
\hline 1976 & 12.637 .962 & 259.237 & 2,05 & 2.537 .581 & 258.853 & 10,20 & 99,85 \\
\hline 1977 & 16.141 .762 & 230.723 & 1,43 & 1.652 .570 & 220.187 & 13,32 & 95,43 \\
\hline 1978 & 4.598 .596 & 215.710 & 4,69 & 1.483 .122 & 205.646 & 13,87 & 95,33 \\
\hline 1979 & 4.104.971 & 178.415 & 4,35 & 1.004 .149 & 169.492 & 16,88 & 95,00 \\
\hline 1980 & 2.064 .226 & 50.747 & 2,46 & 664.246 & 46.758 & 7,04 & 92,14 \\
\hline 1981 & 5.913 .874 & 108.161 & 1,83 & 1.375 .863 & 98.168 & 7,14 & 90,76 \\
\hline 1982 & 5.053 .292 & 228.833 & 4,53 & 1.416 .974 & 217.891 & 15,38 & 95,22 \\
\hline 1983 & 3.846 .018 & 171.515 & 4,46 & 850.149 & 159.103 & 18,71 & 92,76 \\
\hline 1984 & 8.312 .996 & 157.803 & 1,90 & 1.106 .292 & 148.924 & 13,46 & 94,37 \\
\hline 1985 & 5.021 .658 & 402.406 & 8,01 & 1.644 .544 & 384.876 & 23,40 & 95,64 \\
\hline 1986 & 7.131 .761 & 410.880 & 5,76 & 1.737.241 & 364.028 & 20,95 & 88,60 \\
\hline 1987 & 9.163 .928 & 480.545 & 5,24 & 2.132 .863 & 450.625 & 21,13 & 93,77 \\
\hline 1988 & 14.005 .477 & 372.741 & 2,66 & 2.237 .412 & 352.033 & 15,73 & 94,44 \\
\hline 1989 & 9.713 .836 & 228.964 & 2,36 & 2.068 .148 & 220.523 & 10,66 & 96,31 \\
\hline 1990 & 9.202 .507 & 181.983 & 1,98 & 2.507 .226 & 176.647 & 7,05 & 97,07 \\
\hline 1991 & 14.404 .961 & 232.666 & 1,62 & 1.739 .952 & 212.399 & 12,21 & 91,29 \\
\hline
\end{tabular}

(1) \% de inversión sectorial de la provincia de Alicante sobre el calzado de la Comunidad Valenciana. 


\section{Cuadro $\mathrm{V}$}

RELACIÓN DE LA INVERSIÓN CON OTROS FACTORES PRODUCTIVOS EN LA PROVINCIA DE ALICANTE

\begin{tabular}{|r|rrrr|rrrr|}
\hline & \multicolumn{3}{|c|}{ TOTAL SECTOR CALZADO } & \multicolumn{5}{c|}{ TOTAL INDUSTRIAL } \\
\hline & Inversión & Empleo & Potencia & Inscripción & Inversión & Empleo & Potencia & Inscripción \\
\hline 1975 & 112.566 & 821 & 1.066 & 73 & 2.129 .477 & 4.297 & 38.533 & 676 \\
1980 & 46.758 & 758 & 1.407 & 73 & 664.246 & 1.852 & 10.620 & 448 \\
1985 & 384.876 & 4.367 & 12.331 & 384 & 1.644 .544 & 6.921 & 45.277 & 1.158 \\
1991 & 212.399 & 1.608 & 6.280 & 140 & 1.739 .952 & 3.829 & 39.324 & 660 \\
\hline
\end{tabular}

\begin{tabular}{|r|rr|rr|rc|cr|}
\hline & \multicolumn{3}{|c|}{ TOTAL SECTOR CALZADO } & \multicolumn{4}{c|}{ TOTAL INDUSTRIAL } \\
\hline & \multicolumn{2}{|c|}{ Inversión/ } & \multicolumn{2}{|c|}{ Potencia (KW) } & \multicolumn{2}{c|}{ Inversión/ } & \multicolumn{2}{|c|}{ Potencia (KW) } \\
\hline & Empleo & Inscripción & Empleo & Inscripción & Empleo & Inscripción & Empleo & Inscripción \\
\hline 1975 & 137 & 1.542 & 1 & 15 & 496 & 3.150 & 9 & 57 \\
1980 & 62 & 641 & 2 & 19 & 359 & 1.483 & 6 & 24 \\
1985 & 88 & 1.002 & 3 & 32 & 238 & 1.420 & 7 & 39 \\
1991 & 132 & 1.517 & 4 & 45 & 454 & 2.636 & 10 & 60 \\
\hline
\end{tabular}

Valores de la inversión en miles de pesetas constantes (74).

Cuadro VI

DISTRIBUCIÓN DE LA INVERSIÓN INDUSTRIAL TOTAL EN LA PROVINCIA DE ALICANTE

\begin{tabular}{|c|c|c|c|c|c|c|c|c|c|c|}
\hline & \multicolumn{5}{|c|}{$\begin{array}{l}\text { INVERSIÓN EN PORCENTAJES: } \\
\text { SECTOR CALZADO }\end{array}$} & \multicolumn{5}{|c|}{$\begin{array}{c}\text { INVERSIÓN EN PORCENTAJES: } \\
\text { TOTAL INDUSTRIAL }\end{array}$} \\
\hline & \multicolumn{3}{|c|}{ Maquinaria } & \multirow{2}{*}{$\begin{array}{l}\text { Infraes- } \\
\text { tructura }\end{array}$} & \multirow[t]{2}{*}{ Otras } & \multicolumn{3}{|c|}{ Maquinaria } & \multirow{2}{*}{$\begin{array}{l}\text { Infraes- } \\
\text { tructura }\end{array}$} & \multirow[t]{2}{*}{ Otras } \\
\hline & Nacional & Importada & Total & & & Nacional & Importada & Total & & \\
\hline 1975 & 52 & 48 & 81 & 16 & 3 & 79 & 21 & 75 & 20 & 5 \\
\hline 1980 & 88 & 12 & 82 & 14 & 4 & 65 & 35 & 63 & 33 & 4 \\
\hline 1985 & 70 & 30 & 83 & 11 & 6 & 79 & 21 & 74 & 22 & 4 \\
\hline 1991 & 77 & 23 & 63 & 26 & 11 & 69 & 31 & 67 & 28 & 5 \\
\hline
\end{tabular}

Fuente: Consellería d'Indústria, Comerç i Turisme.

Con la crisis de los ochenta muchas de las empresas del sector, PYMEs, con escasos recursos financieros, se enfrentaron a la demanda de dinero que requería la renovación del utillaje o la incorporación de nuevas técnicas y diseños, acudiendo al crédito privado de corta duración, y a la autofinanciación. La ayuda pública del plan de reconversión no funcionó bien. Pero organismos como INESCOP o FICIA permitieron paliar en parte esta deficiencia facilitando una serie de servicios. 
Las empresas que continuaron, con la incorporación a la CEE, tuvieron que aumentar las inversiones ante la necesidad de adaptarse a la competencia internacional, en concreto la italiana. Muchas empresas renovaron sus máquinas e infraestructura, pero se encontraron con una disminución de las exportaciones.

Los datos de inversión indican (cuadro VI):

a) Que la maquinaria es de procedencia nacional en su mayor parte, aunque esta afirmación debería matizarse si tuviésemos en cuenta las patentes y otras circunstancias como la recompra de las empresas que cierran o la utilización de maquinaria procedente del cierre de un establecimiento anterior.

b) Que la inversión en maquinaria fue aproximadamente el $80 \%$ del total durante todo el periodo excepto en 1991 cuando los gastos en infraestructura y otros aumentaron.

c) Que los gastos en infraestructura y otros, debidos a la necesidad de adaptarse a la legislación urbanística, laboral y de impacto ambiental, son cada vez mayores.

Dos aspectos aludidos, tecnología e infraestructura constituyen, respectivamente, la segunda y tercera preocupación de los empresarios dentro de los factores de producción. Se considera que sus costes son elevados y que sólo los superan los gastos laborales.

Por último, una cuestión a considerar en los factores que influyen en la localización de las inversiones es su procedencia. La mayor parte del capital procede de la reinversión, que por otra parte se halla muy mermado por los problemas de la lentitud en cobrar o por los impagados. El crédito privado no resulta difícil de conseguir, pero los altos costes de los intereses en la década de los ochenta e inicios de los noventa suponen un fuerte freno. Las ayudas oficiales se solicitan en general por las grandes empresas que tienen mayores medios para superar las trabas burocráticas, pero no por las pequeñas y medianas empresas que ven en los procedimientos administrativos un fuerte obstáculo ${ }^{25}$.

\section{Distribución geográfica de la inversión (1975-1991)}

En la distribución geográfica de la inversión en el sector del calzado debemos tener en cuenta (cuadro VII):

1. La existencia de dos municipios, Elche y Elda, que de forma regular concentran más de la mitad de la inversión.

2. Estos dos municipios cada vez representan un menor porcentaje de la inversión provincial del sector.

3. La difusión espacial se concentra, como se observa en el mapa de 1975, dentro de lo que es el valle del Vinalopó. En 1980, coincidiendo con la mayor crisis del periodo analizado y pese a la reducción del valor de la inversión, se produce una dispersión por las zonas próximas colindantes.

4. En 1985, en una fase álgida, continúa la dispersión geográfica afectando ya a municipios relativamente alejados del Valle del Vinalopó como Denia y Calpe.

5. Este movimiento centrífugo acaba afectando directamente a la capital de provincia en 1991.

En un análisis global de la distribución geográfica de la inversión durante el periodo se puede concluir: 


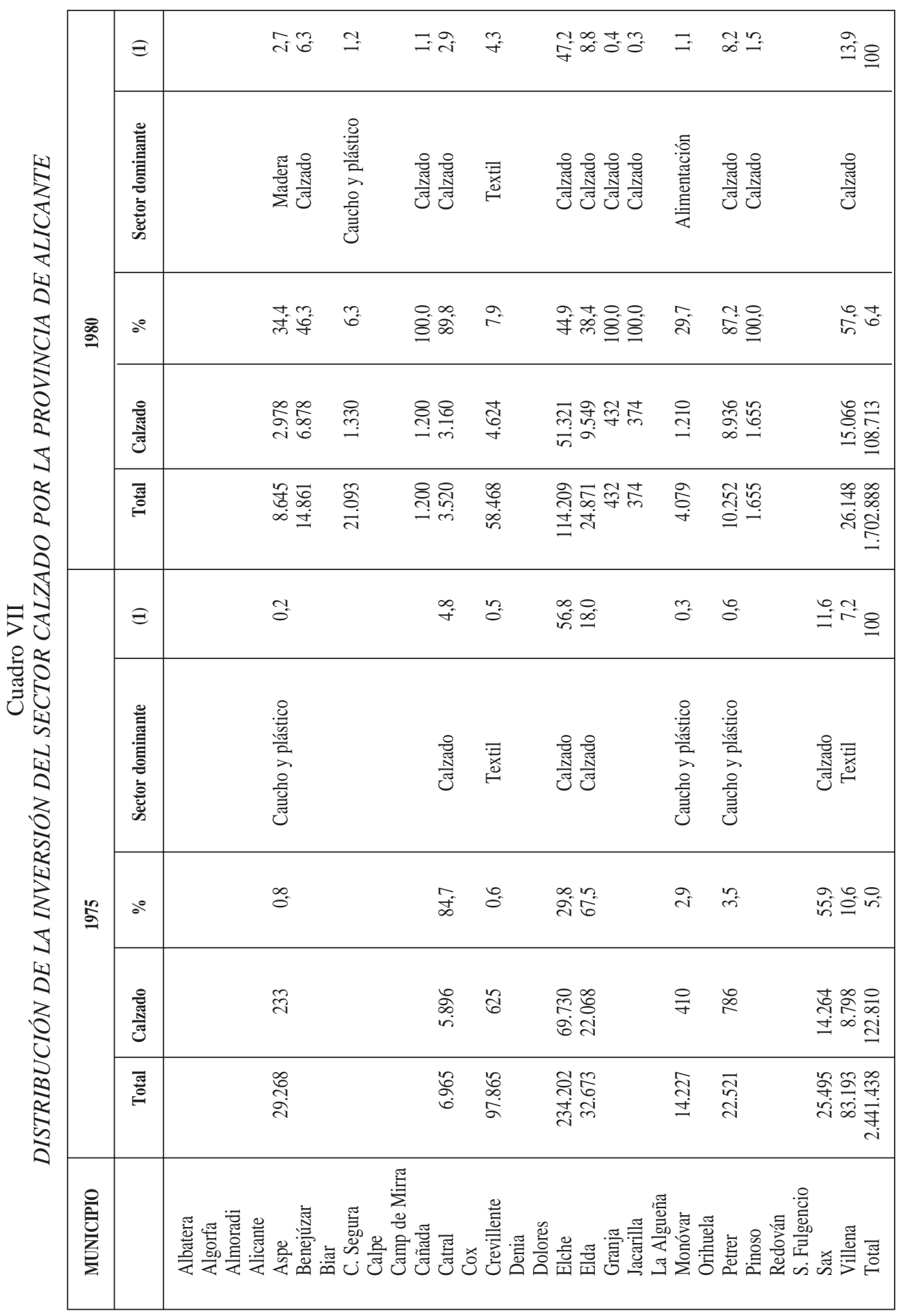




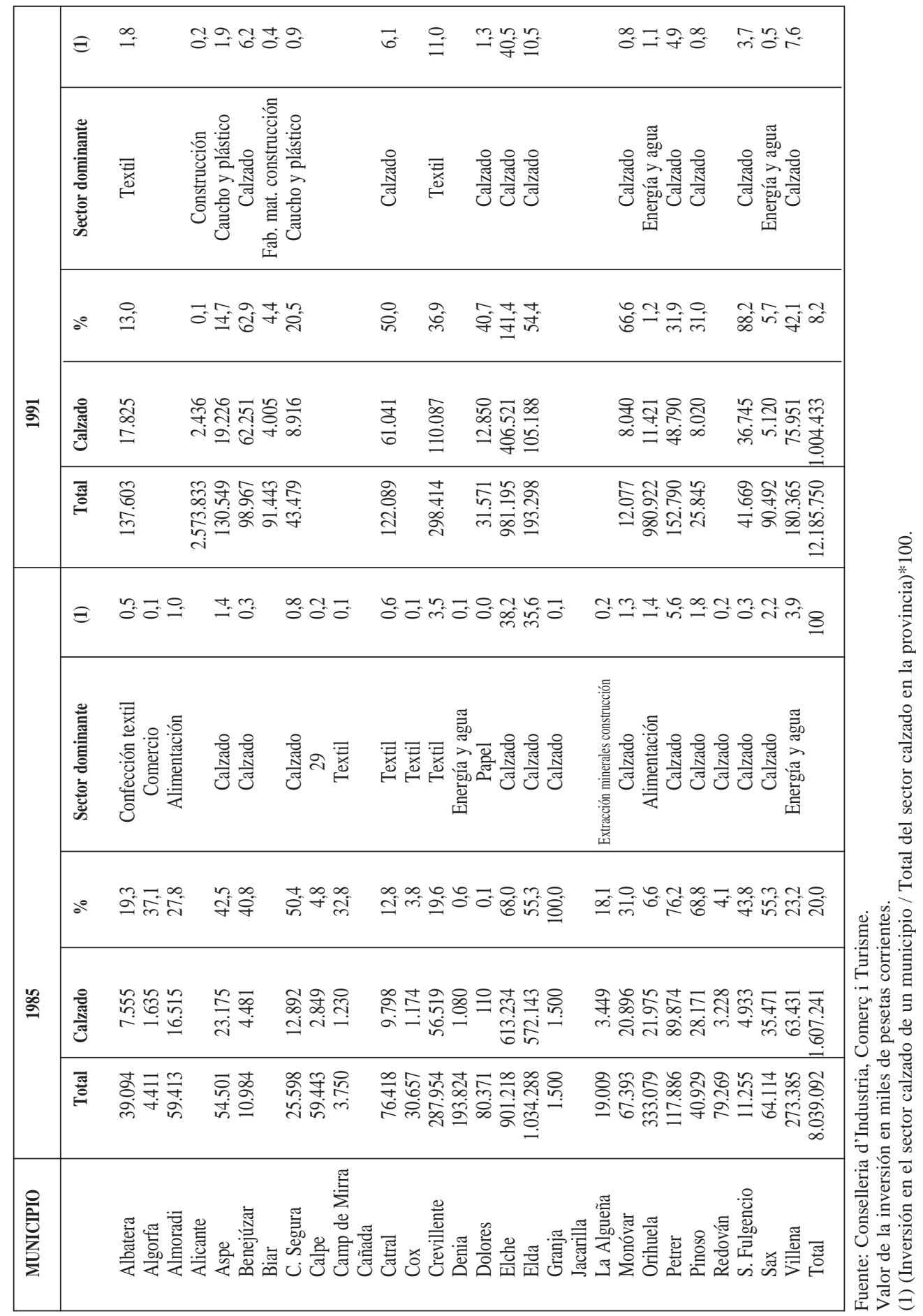


a) Que la difusión de la inversión en la industria del calzado se realiza en función de la rentabilidad de las actividades existentes en cada municipio:

- En los municipios agrícolas próximos al Bajo y Medio Vinalopó la actividad del calzado se introdujo sin problemas desde un primer momento.

- En los municipios turísticos del litoral, tanto del Bajo Vinalopó como de la Vega Baja, en los cuatros años seleccionados no recibieron ninguna inversión.

- Los municipios con tradición industrial y que se emplazaban dentro de un «distrito industrial», frenaron el avance del sector calzado; así ocurre con el textil y el juguete en las proximidades del Alto y Medio Vinalopó.

— Los municipios más alejados dedicados a servicios, o que localizaban alguna industria endógena como Alicante, Agost o Jijona, apenas recibieron inversión del sector.

b) En las zonas más próximas a los núcleos vertebradores del «distrito industrial del calzado», existen municipios donde la competencia entre los distintos sectores es importante y depende no tanto de la tradición sino de la coyuntura económica por la que atraviesan éstos. De este modo, en 1991, el calzado todavía no consiguió ser hegemónico en Crevillente, aunque le faltó poco para alcanzar al textil en la inversión, no así en los puestos de trabajo previsto en los que sí le superó. En Villena, el sector del calzado consiguió desplazar al textil y en Aspe el sector de la madera fue superado por el calzado; en Monóvar, Almoradí y Orihuela ocurrió lo mismo con el de la alimentación. 